\title{
Efeitos do ensino por problemas sobre a atividade física e aptidão física em escolares
}

\author{
Effects of problem-solving teaching on physical activity and physical fitness in \\ schoolchildren
}

\section{AUTORES \\ Ademir Testa Junior ${ }^{1}$ (D) \\ Idico Luiz Pellegrinotti ${ }^{2}$ (D) \\ 1 Faculdades Integradas de Jaú, Departamento de \\ Educação Física, Jaú, São Paulo, Brasil. \\ 2 Universidade Metodista de Piracicaba, \\ Departamento de Pós-graduação em Ciências no \\ Movimento Humano, Piracicaba, São Paulo, Brasil.}

\section{CONTATO}

Ademir Testa Junior

ademirtj@gmail.com

Rua Orsolo D’Andrea, 131, Residencial

Bernardi, Jaú, São Paulo, Brasil.

CEP: $17210-832$.

DOI

$10.12820 /$ rbafs. $24 \mathrm{e} 0110$

\section{(cc) BY-NC-SA}

Este obra está licenciado com uma Licença Creative Commons Atribuição-NãoComercialCompartilhaIgual 4.0 Internacional

\begin{abstract}
RESUMO
O objetivo do estudo foi analisar as mudanças na atividade física e aptidão física após aulas de Educação Física escolar fundamentadas no ensino por resolução de problemas. Participaram da pesquisa, dois grupos (G1 n = 33 e G2 n=35) compostos por jovens com 17 anos de idade. Os integrantes do G1 e do G2 participaram de aulas sobre conteúdo da aptidão física, por meio da metodologia baseada na resolução de problemas, durante 20 aulas. Os integrantes do G1 tiveram as aulas predominantemente teóricas; para o $\mathrm{G} 2$, as aulas foram predominantemente práticas. A bateria de testes de aptidão física da PROESP-BR e o International Physical Activity Questionnaire (IPAQ-versão curta) foram utilizados antes e após a intervenção. A quantidade de participantes muito ativos aumentou $21,2 \%$ no G1 e não sofreu alterações no G2 $(31,4 \%)$. Houve diferença significante $(\mathrm{p}<0,05)$ entre momentos (pré vs pós) no G1, na flexibilidade e força abdominal, e efeito pequeno para a flexibilidade, velocidade e força abdominal. No G2 observou-se diferença significante e efeito pequeno para flexibilidade, resistência e velocidade. Concluiu-se que as duas intervenções didáticas promoveram alteração no nível de atividade física, mas o efeito foi maior nos escolares do G1. O nível de aptidão física foi maior após as intervenções no G1 e no G2. O estudo revelou a importância de se promover a disseminação dos saberes e valores sobre a vida fisicamente ativa.
\end{abstract}

Palavras-chave: Exercício físico; Aptidão física; Ensino; Estilo de vida sedentário; Resolução de problema.

\begin{abstract}
The aim of the study was to analyze changes in physical activities and physical fitness after school Physical Education classes based on the methodology based on teaching by problem solving. Participated in the study, 2 groups (G1 $n=33$ and G2 n= 35) composed of students aged 17 years old. The members of G1 and G2 participated in classes on physical fitness content, through a methodology based on problem solving, during 20 classes. The members of G1 had classes that were predominantly theoretical; for G2, the classes were predominantly practical. PROESP-BR's battery of physical fitness tests and the International Physical Activity Questionnaire (IPAQ-short version) were used before and after the intervention. The number of very active participants increased $21.2 \%$ in G1 and did not change in G2 (31.4\%). There was a significant difference $(p<0.05)$ between moments (pre vs post) in G1, in flexibility and abdominal strength, and small effect for flexibility, speed and abdominal strength. In G2 there was a significant difference and small effect for flexibility, resistance and speed. It was concluded that the two didactic interventions promoted changes in the level of physical activity, but the effect was greater in the students of G1. The level of physical fitness was bigher after interventions in $G 1$ and $G 2$. The study revealed the importance of promoting the dissemination of knowledge and values about physically active life.
\end{abstract}

Keywords: Physical exercise; Physical fitness; Teaching; Sedentary lifestyle; Problem solving.

\section{Introdução}

As evidências sobre a inatividade física na população jovem não são recentes. Um estudo realizado em 2000 com adolescentes de Niterói, Rio de Janeiro mostrou que $33,6 \%$ dos meninos e $52,5 \%$ das meninas foram classificados como muito inativos fisicamente, e $51,2 \%$ dos meninos e $41,6 \%$ das meninas como inativos fisicamente ${ }^{1}$. Estudo em Florianópolis, Santa Catarina ${ }^{2}$ e Aracajú, Sergipe $^{3}$, realizados em 2006 e 2015, já observavam que
$62,2 \%$ e $81,9 \%$ dos jovens eram considerados fisicamente inativos, respectivamente. Atualmente, a Organização Mundial da Saúde coloca o Brasil entre os países com maior índice de inatividade física do mundo ${ }^{4}$.

Diante desse cenário, intervenções têm considerado as aulas de educação física como um contexto potencial para a promoção do estilo de vida fisicamente ativo ${ }^{5}$. Contudo, intervenções que propuseram o aumento da prática de atividades físicas nas aulas de educação fí- 
sica, alterações no espaço escolar, atividades realizadas durante os intervalos das aulas ou atividades em salas de aula não têm mostrado alterações significantes na prática de atividades físicas ou a manutenção dos resultados positivos ${ }^{6}$.

Admitir a saúde como objetivo geral da Educação Física escolar ${ }^{7}$ ou entendê-la como tema relevante atualmente não garante o aumento dos saberes e dos comportamentos saudáveis na população escolar. No entanto, as aulas de Educação Física são os momentos específicos e adequados para o desenvolvimento de ações pedagógicas, intencionalmente organizadas para a construção de aprendizagens significantes, sobre a importância da vida fisicamente ativa em relação à saúde das pessoas e sua relevância social e cultural. Portanto, um desafio à abordagem da saúde durante as aulas de Educação Física na escola reside em promover aprendizagens que possibilitem a tomada de decisão consciente sobre a adoção do estilo de vida ${ }^{8}$, principalmente, considerando que a prática de atividades físicas é uma flutuante e tende a se modificar conforme o contexto e os aspectos envolvidos ${ }^{9}$.

Nesse contexto, há necessidade de se avaliar propostas pedagógicas não convencionais para a abordagem dos conteúdos da atividade física, aptidão física e saúde nas aulas de Educação Física. $\mathrm{O}$ ensino baseado em problemas pode favorecer a motivação para a mudança de comportamento dos escolares, como consequência da compreensão do sedentarismo como óbice social. Como as propostas pedagógicas que problematizam estas temáticas nas aulas de Educação Física são escassas, não se sabe os potenciais de intervenção sobre os componentes de saúde dos escolares.

O presente estudo objetivou analisar se programas de Educação Física com aulas predominantemente teóricas ou práticas, baseados na resolução de problemas, ocasionam mudanças na atividade física e na aptidão física em escolares.

\section{Método}

Esta pesquisa experimental foi realizada após aprovação do Comitê de Ética em Pesquisa através da plataforma Brasil (CAAE: 57279816.2.0000.5430) e respeitou as normas de conduta em pesquisa experimental com seres humanos (Resolução 466/12 do CNS).

Participaram da pesquisa os escolares matriculados na $3^{a}$ série do ensino médio de uma escola pública de Jaú da rede estadual paulista de ensino. A escola e as turmas foram escolhidas a partir da atribuição de aulas do ano de 2016. Eram as únicas duas turmas da $3^{\text {a }}$ do ensino médio, cujos alunos foram convidados a participarem. A definição de qual turma seria G1 e qual seria G2 foi realizada através de sorteio.

A amostra foi composta por dois grupos (G1 - grupo 1 e G2 - grupo 2) de escolares que tinham 17 anos de idade. O G1 foi composto por 33 escolares, sendo $54,54 \%(n=18)$ eram do sexo feminino e $45,5 \%$ $(n=15)$ eram do sexo masculino. A média de massa e estatura corporal entre os participantes do G1 era respectivamente de $66,29 \mathrm{Kg}( \pm 14,02)$ e 1,67 metros $( \pm 0,1)$. Já o $\mathrm{G} 2$ foi formado por 35 participantes, dos quais $42,8 \%(\mathrm{n}=15)$ eram do sexo feminino e $57,1 \%$ $(\mathrm{n}=20)$ eram do sexo masculino. Os integrantes do G2 apresentaram média de massa e estatura de $68,94 \mathrm{Kg}( \pm$ $13,74)$ e 1,69 metros $( \pm 0,09)$ respectivamente.

Para participar da pesquisa, os escolares apresentaram os Termos de Assentimento e Consentimento Livre e Esclarecido assinados por eles e pelos seus responsáveis legais, além de participar de todas as atividades propostas durante as intervenções.

Os participantes dos dois grupos foram envolvidos em atividades pedagógicas durante as suas aulas de educação física, as quais tinham duração de duas aulas de 50 (cinquenta) minutos por semana durante um bimestre letivo, totalizando 20 (vinte) aulas.

Para o G1, as aulas foram predominantemente teóricas, ou seja, compostas por estudos sobre conteúdos da aptidão física. As aulas do G2, que tiveram abordagem dos mesmos conteúdos e sequência didática do G1, apresentaram predominância prática, ou seja, vivências motoras sobre os conteúdos da aptidão física. Tanto a proposta interventiva de predominância teórica quanto a de predominância prática foram aplicadas através da metodologia de resolução de problemas ${ }^{8,10}$.

As aulas teóricas foram realizadas em diferentes espaços da escola, majoritariamente na sala de aula, pois estiveram caracterizadas por momentos de exposição sobre o assunto com uso de slides pelo professor, estudos de textos, rodas de discussão, produção de textos, respostas a questionários, elaboração de relatórios e estratégias para resolução de problemas. As aulas práticas foram realizadas na quadra poliesportiva da escola e organizadas a partir de experiências sobre os conteúdos da aptidão física, envolvendo o planejamento, organização e prática de diferentes tipos de exercícios físicos, avaliação física, aplicação de estratégias para a resolução de problemas.

As sequências foram compostas pelas seguintes eta- 
pas: 1- Identificação do problema: esse momento foi o de ajudar os estudantes a identificarem o problema apresentado pelo professor, reconhecendo-o como problema a ser resolvido. Tal problema deveria estar necessariamente relacionado ao tema estabelecido; 2- Compreensão do problema: nesse momento foi necessário que os estudantes compreendessem o problema, e para tal foi importante que os mesmos realizassem uma coleta de informações e interpretação das mesmas, conceituando cada termo utilizado no problema; 3- Conceber um plano: após a compreensão do problema, foi preciso elaborar um plano para conseguir alcançar possíveis respostas para o problema; 4- Execução do plano: realizar nova coleta e interpretação de dados, bem como análise e inferências sobre as informações coletadas; 5- Elaboração de mapas conceituais: através dos dados coletados, e das inferências realizadas, os estudantes elaboraram desenhos gráficos que explicitassem a lógica presente em seus pensamentos sobre o tema até o momento, ou seja, a compreensão e organização conceitual das informações e até resultados obtidos; 6- Comunicação e visão retrospectiva dos resultados e conclusões: foi a exibição escrita e oral sobre o que os estudantes aprenderam, de maneira argumentativa, explicitando os dados obtidos ${ }^{8,10}$.

Antes e após (agosto e novembro de 2018) a realização das intervenções pedagógicas, foram aplicados os instrumentos de coleta de dados e os testes de aptidão física. Os testes foram aplicados pelo próprio pesquisador na sala de aula (questionários), quadra poliesportiva (testes de aptidão física) e sala da direção escolar (dobras cutâneas), organizados em 5 dias. No $1^{\circ}$ dia foram coletadas as medidas de massa corporal e a estatura, no $2^{\circ}$ dia a aplicação do questionário de atividade física, no $3^{\circ}$ dia a medida das dobras cutâneas e nos $4^{\circ}$ e $5^{\circ}$ dias a realização dos testes de aptidão física. A aplicação do questionário foi iniciada com a apresentação de instruções sobre o instrumento e foram sanadas todas as dúvidas relacionadas às questões durante o seu preenchimento. As medidas de dobras cutâneas foram coletadas com a presença de cinco participantes por vez. Os testes de aptidão física foram organizados em dois dias, sendo o primeiro dedicado à realização dos testes de flexibilidade, resistência cardiorrespiratória, potência muscular dos membros inferiores e membros superiores, e o segundo dia aos testes de agilidade, velocidade e força abdominal. Os testes de flexibilidade, potência muscular dos membros inferiores e superiores, além da agilidade foram aplicados em duas tentativas e considerada a melhor marca em cada teste. Cuidou-se para que cada participante tivesse pelo menos $10 \mathrm{mi}-$ nutos de descanso entre a execução de cada teste.

O International Physical Activity Questionnaire (IPAQ) em sua versão curta foi aplicado considerando que as aulas de Educação Física de um grupo seriam fisicamente mais ativas em relação ao outro, por isso foi solicitado aos participantes que não considerassem o tempo das aulas de Educação Física ao responderem o questionário (IPAQ). Então o resultado do IPAQ caracterizou-se pela representação do nível de prática de atividade física dos participantes, exceto as atividades realizadas durante as aulas de Educação Física na escola. As respostas dos participantes para as questões do IPAQ foram avaliadas segundo o instrumento para classificação do nível de atividade física conforme instrumento para avaliação dos resultados do IPAQ, o que permitiu classificar os participantes em inativos, irregularmente ativos, ativos e muito ativos ${ }^{11}$.

Para a avaliação do percentual de gordura corporal utilizou-se o protocolo de duas dobras cutâneas de Slauther ${ }^{12}$, para jovens de 8 a 18 anos de idade. Foi utilizada a média entre 3 medidas coletadas com adipômetro clínico da marca Sanny ${ }^{\circledR}$, cujas medidas foram em milímetros.

Os testes de aptidão física foram aplicados seguindo o protocolo da PROESP-BR. Para aferição dos níveis de flexibilidade os participantes estavam descalços, considerando-se a melhor marca em centímetros no teste de sentar e alcançar. A resistência cardiorrespiratória foi medida em metros percorridos em 6 minutos em um percurso retangular com 30 metros de lado e 15 metros de fundo. A potência muscular dos membros inferiores foi medida a partir da distância do salto horizontal em centímetros, partindo da posição parada, e membros superiores a partir da distância, em centímetros, do arremesso da medicine ball de dois quilos. A agilidade foi verificada pelo tempo, em segundos e centésimos de segundos, que os participantes concluíram o percurso no quadrado de 4 metros de lado. A velocidade foi medida pelo tempo, em segundos e centésimos de segundos, que os alunos percorreram os 20 metros do teste na maior rapidez possível. A resistência abdominal foi determinada pelo número máximo de repetições realizadas em um minuto ${ }^{13}$.

A análise dos dados foi realizada através do corte quantitativo, identificando e comparando os valores em ambos os grupos para o nível de atividade física e de aptidão física dos escolares. 
As diferenças de proporção nas classificações dos momentos pré e pós do nível de atividade física foram analisadas através do teste qui-quadrado de Pearson $\left(\mathrm{X}^{2}\right)$.

Os dados sobre as variáveis da aptidão física foram analisados por meio de técnicas estatísticas descritivas (média e desvio padrão). Inicialmente foi analisada a normalidade dos dados pelo teste de Shapiro-Wilk e homocedasticidade (critério Bartlett) para identificar as características dos dados. Foi utilizado ANOVA medidas repetidas de dois fatores para a análise da variância entre o fator intervenção (aulas práticas vs. aulas teóricas) e o fator tempo (pré vs. pós intervenção). Em adição, o tamanho do efeito (d Cohen) de cada variável foi calculado usando a diferença da média pré e pós dividida pela média do desvio padrão. Para a classificação do efeito, os valores considerados foram: $<0,2$ - trivial, $\geq 0,2$ e $<0,6$ - pequeno, $\geq 0,6$ e $<1,2-$ moderado, $\geq 1,2$ e $<2,0$ - grande, $\geq 2,0$ e $<4,0$ muito grande e $\geq 4,0-$ extremamente grande ${ }^{14}$.

Utilizou-se o software Graphpad Prism (versão 6.01, Prism for Windows, setembro 21, 2012). O nível de significância adotado foi de $\mathrm{p}<0,05$.

\section{Resultados}

A Figura 1 mostra que, entre os integrantes do G1, a quantidade de participantes classificados como muito ativos aumentou $21,2 \%$ após a aplicação da proposta didática, a quantiadade de ativos e irregularmente ativos diminuiu $15,1 \%$ e $9,1 \%$ respectivamente, e os inativos aumentaram $3,0 \%$.

Já entre os integrantes do G2, o número de classificados como muito ativos não apresentou alterações $(31,4 \%)$, os ativos tiveram uma elevação de $5,7 \%$, irregularmente ativos ou inativos apresentaram declínio no percentual $(\Delta=2,86 \%)$, após a aplicação da intervenção.

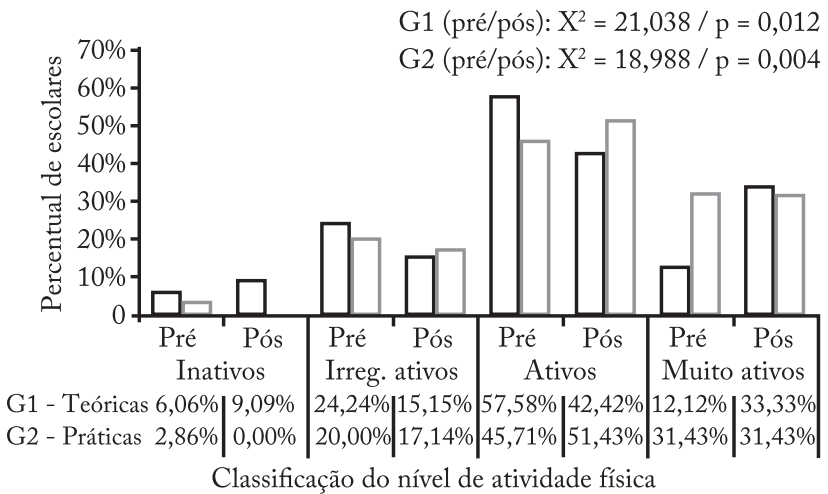

Figura 1 - Percentual de escolares classificados segundo o IPAQ $\mathrm{G} 1$ = grupo $1 ; \mathrm{G} 2$ = grupo $2 ; \mathrm{X}^{2}=$ Qui-quadrado; $\mathrm{p}=$ significância; Pré $=$ antes da aplicação da intervenção; Pós = após a aplicação da intervenção; $\%$ = percentual; Irregul. ativos = irregularmente ativos.

As diferenças de proporções nas classificações do nível de atividade física, nos momentos pré e pós, foram significantes $(p<0,05)$ no G1 e no G2.

Como mostra Tabela 1, para os participantes do G1, houve diferença significante $(\mathrm{p}<0,05)$ entre momentos (pré vs pós) na flexibilidade e força abdominal. Além disso detectou-se que o efeito foi considerado pequeno para a flexibilidade, velocidade e força abdominal.

No G2 foi encontrada diferença significante ( $\mathrm{p}<$ $0,05)$ e efeito pequeno para flexibilidade, resistência e velocidade.

Comparando a média da diferença percentual de cada variável da aptidão física entre o G1 e o G2, cujos dados são exibidos na Tabela 2, observou-se diferença significante na interação tempo vs. intervenção ( $\mathrm{p}<$ $0,05)$ sobre a força abdominal, indicando que as maiores mudanças foram observadas no G1. Efeitos peque-

Tabela 1 - Comparação intragrupos das médias $(\bar{x})$ e desvios padrões (dp) das variáveis da aptidão física, antes e após a aplicação da intervenção

\begin{tabular}{|c|c|c|c|c|c|c|c|c|c|c|}
\hline \multirow{2}{*}{ Variável } & \multicolumn{4}{|c|}{ G1 $(n=33)$} & \multicolumn{6}{|c|}{$\mathrm{G} 2(\mathrm{n}=35)$} \\
\hline & Pré $(\bar{x}, d p)$ & 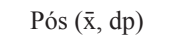 & $\mathrm{p}$ valor & $d$ & Cla. d & Pré $(\bar{x}, d p)$ & Pós ( $\bar{x}, d p)$ & $\mathrm{p}$ valor & $\mathrm{d}$ & Cla. d \\
\hline Flexibilidade & $33,21 \pm 11,28$ & $37,27 \pm 11,95$ & $0,001^{*}$ & 0,37 & Peq. & $40,89 \pm 12,37$ & $47,69 \pm 12,17$ & $0,000^{*}$ & 0,55 & Peq. \\
\hline Resistência & $751,39 \pm 288,91$ & $801,12 \pm 290,92$ & 0,244 & 0,17 & Trivial & $883,46 \pm 253,83$ & $971,14 \pm 297,48$ & $0,012^{*}$ & 0,32 & Peq. \\
\hline Força MI & $150,94 \pm 41,32$ & $157,21 \pm 45,12$ & 0,325 & 0,15 & Trivial & $158,57 \pm 38,89$ & $166,66 \pm 48,18$ & 0,142 & 0,19 & Trivial \\
\hline Força MS & $422,73 \pm 104,07$ & $416 \pm 111,77$ & 0,694 & 0,06 & Trivial & $449,71 \pm 118,72$ & $454,66 \pm 131,83$ & 0,975 & 0,04 & Trivial \\
\hline Agilidade & $7,96 \pm 1,45$ & $7,99 \pm 1,67$ & 0,996 & 0,02 & Trivial & $6,66 \pm 1,05$ & $6,86 \pm 1,74$ & 0,684 & 0,15 & Trivial \\
\hline Velocidade & $4,53 \pm 0,80$ & $4,73 \pm 1$ & 0,195 & 0,22 & Peq. & $4,2 \pm 0,84$ & $4,57 \pm 1,11$ & $0,005^{*}$ & 0,38 & Peq. \\
\hline Força Abd. & $25,73 \pm 12,70$ & $30,09 \pm 13,28$ & $0,002^{*}$ & 0,34 & Peq. & $30,83 \pm 13,96$ & $29,57 \pm 13,26$ & 0,623 & 0,09 & Trivial \\
\hline$\%$ Gordura & $26,58 \pm 9,72$ & $26,78 \pm 10,76$ & 0,993 & 0,02 & Trivial & $24,85 \pm 10,5$ & $25,73 \pm 11,14$ & 0,327 & 0,08 & Trivial \\
\hline
\end{tabular}

G1 = grupo 1; G2 = grupo 2; $\mathrm{n}$ = número de participantes; Pré = antes da aplicação da intervenção; Pós = após a aplicação da intervenção; $\overline{\mathrm{x}}$ = média $; \mathrm{dp}=$ desvio padrão $\mathrm{MI}=$ membros inferiores; $\mathrm{MS}=$ membros superiores; $\mathrm{Abd} .=$ abdominal; $\%=$ percentual; ${ }^{*} \mathrm{p}<0,05=$ diferença significante; $\mathrm{d}$ = tamanho do efeito; Cla. = classificação; Peq. = pequeno. 
nos e não-significativos $(p>0,05)$ foram observados na flexibilidade, força em membros superiores, velocidade, força abdominal e percentual de gordura.

Tabela 2 - Comparação intergrupos das médias $(\overline{\mathrm{x}})$ e desvios padrões (dp) dos percentuais das diferenças das variáveis da aptidão física, antes e após a aplicação da intervenção

\begin{tabular}{lccccc}
\hline Variável & $\begin{array}{c}\mathrm{G} 1(\mathrm{n}=33) \\
\Delta \%(\overline{\mathrm{x}}, \mathrm{dp})\end{array}$ & $\begin{array}{c}\mathrm{G} 2(\mathrm{n}=35) \\
\Delta \%(\overline{\mathrm{x}}, \mathrm{dp})\end{array}$ & $\begin{array}{c}\mathrm{p} \\
\text { valor }\end{array}$ & $\mathrm{d}$ & $\begin{array}{c}\text { Classif. } \\
\mathrm{d}\end{array}$ \\
\hline Flexibilidade & $16,17 \pm 26,86$ & $22,35 \pm 35,42$ & 0,087 & 0,20 & Pequeno \\
Resistência & $12,43 \pm 34,13$ & $11,82 \pm 25,65$ & 0,403 & 0,02 & Trivial \\
Força MI & $4,51 \pm 14,07$ & $4,41 \pm 17,83$ & 0,772 & 0,06 & Trivial \\
Força MS & $1,89 \pm 5,53$ & $0,91 \pm 12,08$ & 0,251 & 0,29 & Pequeno \\
Agilidade & $0,86 \pm 15,63$ & $2,79 \pm 18,15$ & 0,552 & 0,11 & Trivial \\
Velocidade & $4,53 \pm 14,95$ & $9,15 \pm 18,17$ & 0,294 & 0,28 & Pequeno \\
Força Abd. & $30,28 \pm 57,66$ & $3,33 \pm 34,94$ & $0,002^{*}$ & 0,58 & Pequeno \\
\% Gordura & $0,26 \pm 9,98$ & $5,13 \pm 18,36$ & 0,420 & 0,34 & Pequeno \\
\hline
\end{tabular}

$\mathrm{G} 1$ = grupo $1 ; \mathrm{G} 2$ = grupo $2 ; \mathrm{n}=$ número de participantes; $\overline{\mathrm{x}}=$ média $; \mathrm{dp}=$ desvio padrão; $\mathrm{MI}=$ membros inferiores; $\mathrm{MS}=$ membros superiores; Abd. $=$ abdominal; $\%=$ percentual; ${ }^{*} p<0,05=$ diferença significante; $\mathrm{d}$ = tamanho do efeito, Classif. $=$ classificação .

\section{Discussão}

Os principais achados do presente estudo foram o aumento no nível de atividade física no G1 e G2, com ênfase entre os escolares que tiveram aulas predominantemente teóricas no processo de intervenção, revelando a importância da compreensão sobre os conteúdos da aptidão física na adoção do estilo de vida fisicamente ativo.

As elevações observadas entre a quantidade de escolares classificados como muito ativos no $\mathrm{G} 1(\Delta=21,21 \%)$ e no $\mathrm{G} 2(\Delta=5,71 \%)$ indicaram que a metodologia baseada na resolução de problemas, aplicada tanto com estratégias teóricas quanto práticas, permitiu mudanças no nível de atividade física. Destaca-se que o efeito da proposta sobre o nível de atividade física é maior quando os escolares são conscientizados sobre a importância da prática de atividades físicas, uma vez que pode auxiliar em uma manutenção dos comportamentos ${ }^{15}$.

Apesar da predominância do aumento do nível de atividade física ter ocorrido no G1, as intervenções apresentaram potencial para promover alterações atitudinais por parte dos jovens sobre o nível da prática de atividades físicas, o que corrobora com as ideias de outros estudos que observaram que a participação nas aulas de Educação Física e a intervenção do professor favorecem o aumento da prática de atividades físicas fora do período de aulas escolares ${ }^{5,6,16}$.

Um estudo mostrou que aulas de ginástica foram capazes de promover mudanças no nível de atividade física praticado dentro da escola ${ }^{17}$. No entanto, não se sabe se tal proposta de trabalho promoveu mudanças no comportamento cotidiano dos jovens fora da escola e nem se os efeitos positivos foram duradouros ${ }^{6}$. É a predominância do conteúdo esporte que limita a influência das aulas de Educação Física sobre o nível de atividade física praticado fora do ambiente escolar ${ }^{17}$.

Até o momento não foram encontradas metodologias eficazes para atender as expectativas da tendência da educação para a saúde ${ }^{18}$. No entanto, como existe uma relação positiva entre as intervenções sobre os conteúdos da aptidão física e o nível de atividade física em escolares, considerou-se que a metodologia baseada na resolução de problemas pode ser um caminho interessante no enfrentamento ao desafio de estimular os jovens à vida fisicamente ativa.

Apesar das mudanças detectadas no nível de atividade física dos escolares, a quantidade de jovens irregularmente ativos ou inativos ainda é preocupante ${ }^{2,19}$. Inclusive o nível de atividade física é uma variável flutuante, ou seja, não é estável em contextos diferentes 9 .

Não praticar exercícios físicos mesmo após compreender as implicações da inatividade física no organismo humano implica em considerar que a conversão das aprendizagens conceituais e procedimentais em condutas e atitudes cotidianas depende da tomada de decisão do sujeito, que está relacionada às suas percepções subjetivas em relação às práticas motoras ${ }^{8}$. Porém, o resultado mais expressivo sobre o nível de atividade física detectado no G1 revela a importância das atividades de estudo e das compreensões conceituais para gerar mudanças comportamentais em escolares.

Analisando as diferenças entre as médias dos componentes da aptidão físicas intragrupos, constatou-se que ambos os grupos apresentaram aumento estatisticamente significativo em algumas variáveis, cujo efeito foi pequeno para todas elas. Isso demonstrou que aulas de predominância teórica e fazer atividades físicas fora do ambiente escolar, a partir das aprendizagens adquiridas nas aulas de Educação Física, promoveu efeito semelhante sobre a aptidão física em comparação ao de participar das vivências durante as aulas de Educação Física na escola.

De maneira semelhante ao encontrado no presente estudo, outro concluiu que pequenas alterações nas aulas de Educação Física incrementando-as com exercícios físicos podem promover melhoras em algumas capacidades físicas, tais como a flexibilidade e a impulsão vertical ${ }^{20}$.

É possível aumentar a aptidão física dos escolares como consequência da participação nas aulas de Educa- 
ção Física e implementando atividades de enriquecimento curricular (projetos realizados dentro da escola fora do horário normal de aulas), mas é preciso que as atividades sejam estimulantes à todas as capacidades físicas ${ }^{20}$.

Inclusive as variáveis da aptidão física são determinadas por vários fatores contextuais ${ }^{9,21}$, ou seja, os aspectos ambientais, sociais, políticos e econômicos também podem interferir no quanto as pessoas conseguirão aprimorar suas capacidades físicas.

Um hipótese para a vantagem do efeito das aulas teóricas sobre as aulas práticas na força abdominal pode ser a consequência do maior esforço dos participantes na bateria de testes aplicada após a intervenção, pois as aprendizagens adquiridas durante as aulas teóricas promoveram o maior entusiasmo dos escolares ao realizarem a bateria de testes após as intervenções.

As atividades prazerosas nas aulas de Educação Física favorecem a participação dos escolares nas aulas e a satisfação alcançada pelos estudantes a partir das intervenções de ensino podem promover o engajamento dos alunos na educação física ${ }^{15,22}$. No entanto, o presente estudo sugere que a vivência prática nas aulas de Educação Física não garante mudanças na atividade física e em componentes da aptidão física em relação à saúde diferentes dos observados em aulas predominantemente teóricas.

Em conclusão, considerou-se que o desenvolvimento das duas situações didáticas favoreceu o aumento da prática de atividades físicas, mas em indicadores e intensidades diferentes. Vivenciar as atividades físicas é componente importante ao desenvolvimento das percepções dos escolares acerca dos conteúdos que se estuda, mas é relevante que os processos pedagógicos contemplem momentos de estudo, pois é através da aprendizagem dos conhecimentos e valores sobre a prática de atividades físicas que serão incorporadas e prolongadas como hábitos saudáveis por toda a vida de forma intencional e consciente.

Estes achados ajudam a refletir sobre o compromisso social de educar para a saúde do professor de Educação Física na escola, como mediador da aprendizagem significativa sobre uma vida fisicamente ativa e saudável.

\section{Conflito de interesse}

Os autores declaram não haver conflitos de interesse.

\section{Contribuições dos autores}

Testa Junior A, participou da concepção inicial do estudo, coleta de dados, redação e revisão crítica do texto. Pellegrinotti IL, participou da concepção inicial do estudo, redação e revisão crítica do texto.

\section{Agradecimentos}

Agradecimentos à CAPES pela bolsa de estudos junto à Universidade Metodista de Piracicaba, o que possibilitou o desenvolvimento da pesquisa.

\section{Referências}

1. Silva RCR, Malina RM. Nível de atividade física em adolescentes do município de Niterói, Rio de Janeiro, Brasil. Cad Saúde Pública. 2000;16(4):1091-7.

2. Farias Junior JC. Prevalência e fatores de influência para inatividade física em adolescentes. $\mathrm{R}$ Bras Ci e Mov. 2006;14(1):63-70.

3. Silva DAS, Silva RJS. Associação entre prática de atividade física com consumo de frutas, verduras e legumes em adolescentes do Nordeste do Brasil. Rev Paul de Pediatr. 2015;33(2):167-73.

4. Guthold R, Stevens GA, Riley LM, Bull FC. Worldwide trends in insufficient physical activity from 2001 to 2016: a pooled analysis of 358 population-based surveys with 1. 9 million participants. The Lancet Global Health. 2018;6(10):1077-86.

5. Mura G, Rocha NB, Helmich I, Budde H, Machado S, Wegner M, et al. Physical activity interventions in schools for improving lifestyle in European countries. Clin Pract Epidemiol Ment Health. 2015;11(Suppl 1 M5):77-101.

6. Knopp D, Prat IA, Azevedo MR. Intervenções escolares de médio e longo prazo para promoção de atividade física: Revisão sistemática. Rev Bras Ativ Fís Saúde. 2014;19(2):142-52.

7. Neira MG. Educação física: desenvolvendo competências. 3. ed. São Paulo: Phorte, 2009.

8. Testa Junior A, Zuliani SRQA. Metodologia basada en la resolución de problemas aplicada a las classes de Educación Física en la pespectiva de la Educación para la salud. Dissertação de mestrado (Programa de pós-graduação em Educação). Universidad Del Salvador, Buenos Aires, Argentina, 2012.

9. Rauner A, Jekauc D, Mess F, Schmidt S, Woll A. Tracking physical activity in different settings from late childhood to early adulthood in Germany: the MoMo longitudinal study. BMC Public Health. 2015;15(1):391.

10. Pozo JI. A solução de problemas: aprender a resolver, resolver para aprender. São Paulo: Artmed, 1994.

11. Matsudo S, Araújo T, Matsudo V, Andrade D, Andrade E, Oliveira LC, Braggion G. Questinário internacional de atividade física (IPAQ): estudo de validade e reprodutibilidade no Brasil. Rev Bras Ativ Fís Saúde. 2001;6(2):05-18.

12. Charro MA, Bacurau RFP, Navarro F, Pontes Junior, FL. Manual de Avaliação Física. São Paulo: Phorte, 2010.

13. Gaya ACA, Gaya AR. Projeto esporte Brasil: manual de testes e avaliação. Porto Alegre: UFRGS, 2016.

14. Hopkins WG, Marshall SW, Batterham AM, Hanin J. Progressive statistics for studies in sports medicine and exercise science. Med Sci Sports Exerc. 2009;41(1):3-13.

15. Liz CM, Crocetta TB, Silveira Viana M, Brandt R, Andrade A. Aderência à prática de exercícios físicos em academias de ginástica. Motriz. 2010;16(1):181-8.

16. Curran T, Standage M. Psychological Needs and the Quality of Student Engagement in Physical Education: Teachers as Key Facilitators. J Teach Phys Educ. 2017;36(3):262-76.

17. Farias WLS, Freitas JFF, Iwamoto V, Levandoski G, Leonardi TJ. Educação Física escolar nos anos finais do ensino fundamental e a prática de atividades físicas fora da escola. Rev de Educ do Vale do São Francisco. 2017;7(12):163-76. 
18. Oliveira AAB. Metodologias emergentes no ensino da educação física. Rev da Educ Fís. UEM. 1997;1(8):21-7.

19. Petroski EL, Pelegrini A. Associação entre o estilo de vida dos pais e a composição corporal dos filhos adolescentes. Rev Paul Pediatr. 2009; 27(1):48-52.

20. Rexen CT, Ersboll AK, Moller NC, Klakk H, Wedderkopp N, Andersen LB.Effects of extra school-based physical education on overall physical fitness development-the CHAMPS study DK. Scand. J. Med. Sci. Sports. 2015,25(5):706-15.
21. Mello JB, Mello JHP, Vian F, Gaya AR, Gaya AC. A. Associação da aptidão cardiorrespiratória de adolescentes com a atividade física e a estrutura pedagógica da educação física escolar. Rev Bras Ciênc Esporte. 2019;41(4):367-75.

22. Silva RB, Matias TS, Viana MDS, Andrade A. Relação da prática de exercícios físicos e fatores associados às regulações motivacionais de adolescentes brasileiros. Motri. 2012;8(2):8-21.

Recebido: 09/02/2019

Aprovado: 24/07/2020

\section{Como citar este artigo:}

Testa Junior A, Pellegrinotti IL. Efeitos do ensino por problemas sobre a atividade física e aptidão física em escolares. Rev Bras Ativ Fís Saúde. 2019;24:e0110. DOI: 10.12820/rbafs.24e0110 